\title{
OPTIMUM SALINITY LEVEL FOR THE CULTIVATION OF CILIATED PROTOZOA
}

\author{
YUKINORI MUKAI ${ }^{1,2 *}$, SYAFIQAH HANANI MOHD SHAHIDIN ${ }^{3}$ and \\ NORMAWATY MOHMAAD NOOR ${ }^{1,2}$ \\ ${ }^{1}$ Department of Marine Science, Kulliyyah of Science, International Islamic University Malaysia, \\ Jalan Sultan Ahmad Shah, Bandar Indera Mahkota, 25200 Kuantan, Pahang Malaysia \\ ${ }^{2}$ Institute of Oceanography and Maritime Studies (INOCEM), Kulliyyah of Science, \\ International Islamic University Malaysia, Jalan Sultan Ahmad Shah, \\ Bandar Indera Mahkota, 25200 Kuantan, Pahang, Malaysia \\ ${ }^{3}$ Department of Biotechnology, Kulliyyah of Science, International Islamic University Malaysia, \\ Jalan Sultan Ahmad Shah, Bandar Indera Mahkota, 25200 Kuantan, Pahang Malaysia \\ EE-mail: mukai@iium.edu.my
}

Accepted 3 February 2020, Published online 30 June 2020

\begin{abstract}
In the present study, protozoa was cultured in different salinity water $(10 \%, 20 \%$ and $30 \%)$ to determine the optimum salinity level required for the culturing. We examined the densities of protozoa and the ciliates Euplotes spp. in a general culture and Euplotes encysticus Yonezawa in a separate monoculture. Various species of protozoa were observed in the culture water, with a peak density of 30,000-40,000 cells ml-1 on day 4. The density of Euplotes spp. in the culture water increased with elapsed days, peaking on day 3. One-way analysis of variance showed a significant difference in the densities on day 3 , and post-hoc Tukey-Kramer test results showed that the density at 10\% was significantly higher than that at $30 \%$. The E. encysticus monoculture peaked at 5000-6000 cells ml ${ }^{-1}$, but there was no significant difference between the salinity levels. Although our results are not definitive, the lower salinity level appeared most suitable for culturing protozoa. The ciliated protozoa Euplotes spp. in the current study were smaller than $100 \mu \mathrm{m}$, and they may be suitable size as live feed as starter diet for marine fish larvae with small mouth openings.
\end{abstract}

Key words: Protozoa, Euplotes encysticus, starter live feed, ciliates, salinity

\section{INTRODUCTION}

Protozoa is microscopic, single-celled organisms including ciliates. Protozoa plays an important role in microbial food chains in aquatic environments (Figueiredo et al., 2005, 2007; Mieczan, 2007; Montagnes et al., 2010; Zingel et al., 2012). For instance, Fukami et al. (1999) reported that gut contents of fish larvae in Japanese waters using an epifluorescence microscope, and the larvae of only 5 of 52 fish taxa did not consume protozoa, whereas the gut of a majority contained more than 30 individual protozoa in each fish. Protozoa is also important as live feed in the starter diet for seed production for aquaculture, previous studies reported the ciliated protozoa improved larval survival rates (Nagano et al., 2000a, b; Olivotto et

* To whom correspondence should be addressed. al., 2005; Rhodes \& Phelps, 2006). In the early stage of their lives, commercial fish species such as groupers, Napoleon wrasse (mouth size $133 \mu \mathrm{m}$ ) and angelfish (mouth gap size $160 \mu \mathrm{m}$ ) possess relatively small mouth openings (Hagiwara et al., 2014). These species cannot feed on super small type (SS-type) rotifers and therefore select prey smaller than $100 \mu \mathrm{m}$ (Hagiwara et al., 2014). Although relatively fewer studies have been conducted regarding culturing of ciliated protozoa, since ciliated protozoa are small, soft-bodied and nutritionally rich, they reportedly constitute an ideal live feed in the starter diet of marine fish larvae (Das et al., 2012).

Genus Euplotes is one of ciliate, which was used for larval rearing experiment as starter diets (Nagano et al., 2000a,b: Madhu \& Madhu, 2014). Madhu and Madhu (2014) reported Euplotes feeding group showed high survival rates in early larval stage and 
showed the usefulness of Euplotes as starter diets. Groupers, Napoleon wrasse and snappers have small bodies and small mouth openings in the early larval stage (Doi et al., 1997a; Nagano et al., 2000a; Hagiwara et al., 2014). Therefore, seed production of these fish is difficult due to the lack of suitable live starter feed. Kitani (1989) and Mukai et al. (2016) reported an easy method for the culturing of protozoa in saline water, using minced vegetable leaves to cover the entire surface of several litres of culture water in glass basins. Dry fishmeal was placed on the surface of leaves without aeration. After a period of 3-10 days, protozoa blooms were achieved in the basins. The salinity level of culture water is an important factor in the culturing of protozoa, and the present study was conducted to determine an optimum salinity level for culture. The general ciliated protozoa culture (in which the Euplotes spp. were assessed) and the E. encysticus culture were prepared using the almost same methods as Mukai et al. (2016).

\section{MATERIALS AND METHODS}

\section{Materials for cultivation of protozoa}

Previous studies reports used various types of materials to culture ciliated protozoa; yeast and the microalga, Winter and Persoone (1975); lettuce, cabbage and banana peel (Das et al., 2012); coagulated egg yolk and dried lettuce (Curds et al., 1966), vegetable and fish meals Kitani (1989) and Mukai (2016), Culture Selco 3000 for genus Euplotes (Cortes \& Tsuzuki, 2012). In this study, vegetable and fish meal were used to culture protozoa.

Fresh vegetables [napa cabbage (Brassica rapa var. pekinensis (Lour)) and Chinese cabbage (Brassica rapa var. chinensis)] were purchased from the local general market and carefully washed using tap water. Subsequently, they were cut into small pieces $(10-20 \mathrm{~mm})$. Fish meal was prepared using dried anchovies purchased from the local general market. The dried anchovies were sterilised under moderate heat and ground into a powder using a domestic kitchen blender.

\section{Method for cultivation of ciliates}

The experiments of protozoa culture were conducted in the laboratories of Kulliyyah of Science, International Islamic University Malaysia. Seawater was obtained from Institute of Oceanography and Maritime Studies, International Islamic University Malaysia. The protozoa culture was prepared using the same method reported previously (Mukai et al., 2016), except that the aeration was provided to the culture in this study. The temperature of water during cultivation was $26^{\circ} \mathrm{C}-$ $28^{\circ} \mathrm{C}$.

Protozoa culture water at different salinity levels $(10 \%, 20 \%$ and $30 \%)$ was prepared using seawater and de-chlorinated tap water, and $3 \mathrm{~L}$ of saline water was added into a $5 \mathrm{~L}$ water container. Nutrients for culture protozoa were added $2 \%(\mathrm{w} / \mathrm{v})$ fresh vegetables and $0.25 \%(\mathrm{w} / \mathrm{v})$ fish meal. Protozoa culture was conducted with triplicate water containers of each salinity level. In this protozoa culture water, three types of Euplotes were detected. The Euplotes spp. were treated as Euplotes sp1, sp2, and Euplotes encysticus (Mu'izzuddin-Afandi et al., 2018).

Monoculture of Euplotes encysticus was conducted using seed of E. encysticus that has been kept in Laboratory in Kulliyyah of Science. Different salinity levels water was prepared using de-chlorinated tap water and seawater with chlorination and de-chlorination treatment to remove protozoa in the seawater. A monoculture of E. encysticus was also conducted with triplicate in $1 \mathrm{~L}$ beakers, using the same nutrient concentrations used for the protozoa culture. E. encysticus was isolated to make seed of E. encysticus using a pipette under a stereo microscope and transferred to a $500 \mathrm{ml}$ beaker containing autoclaved seawater with added baker's yeast. Seed of E. encysticus was kept in $500 \mathrm{ml}$ beakers with giving $2-3 \mathrm{mg}$ yeast every few days. Protozoa, Euplotes spp. and E. encysticus were counted using a plankton counter (MPC 200 Optical Plastic Plankton counter, Matsunami Glass Ind. Ltd. Kishiwada, Japan). Densities in each culture container or beaker were counted thrice, and a mean value was obtained.

\section{Statistical analysis}

Statistical analysis of protozoa and Euplotes spp was conducted using one-way Anova for days 1, 2, 3, 4 and 6, and the analysis of Euplotes encysticus culture was conducted using KruskalWallis test for days 1 to 6 , since the variance was not homogenate.

\section{RESULTS}

The density of protozoa grown at different salinity levels with elapsed days was observed (Figure 1). Protozoa densities in salinity $10 \%$ peaked on day 3 and that on salinity $20 \%$ and $30 \%$ peaked on day 4 , then gradually decreased. Various species were present in the protozoa culture water, including ciliated protozoa Euplotes spp., Litonotus sp., Tachysoma sp. and Uronema sp. (morphologically identified with size and shape of cell body and cilia distribution (Corliss, 1979). On the basis of size, 


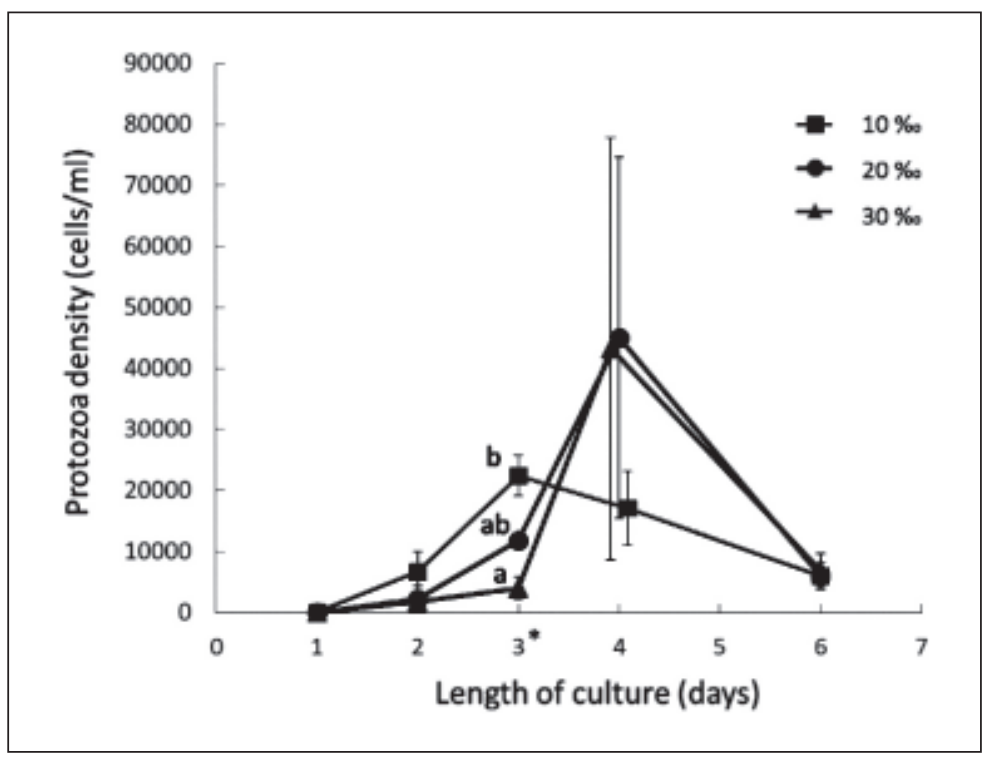

Fig. 1. Protozoa densities (cell $\mathrm{ml}^{-1}$ : mean $\pm \mathrm{SE}$ ) at different salinity levels with elapsed days. $*$ Different lowercase letters indicate a significant difference on day 3 .

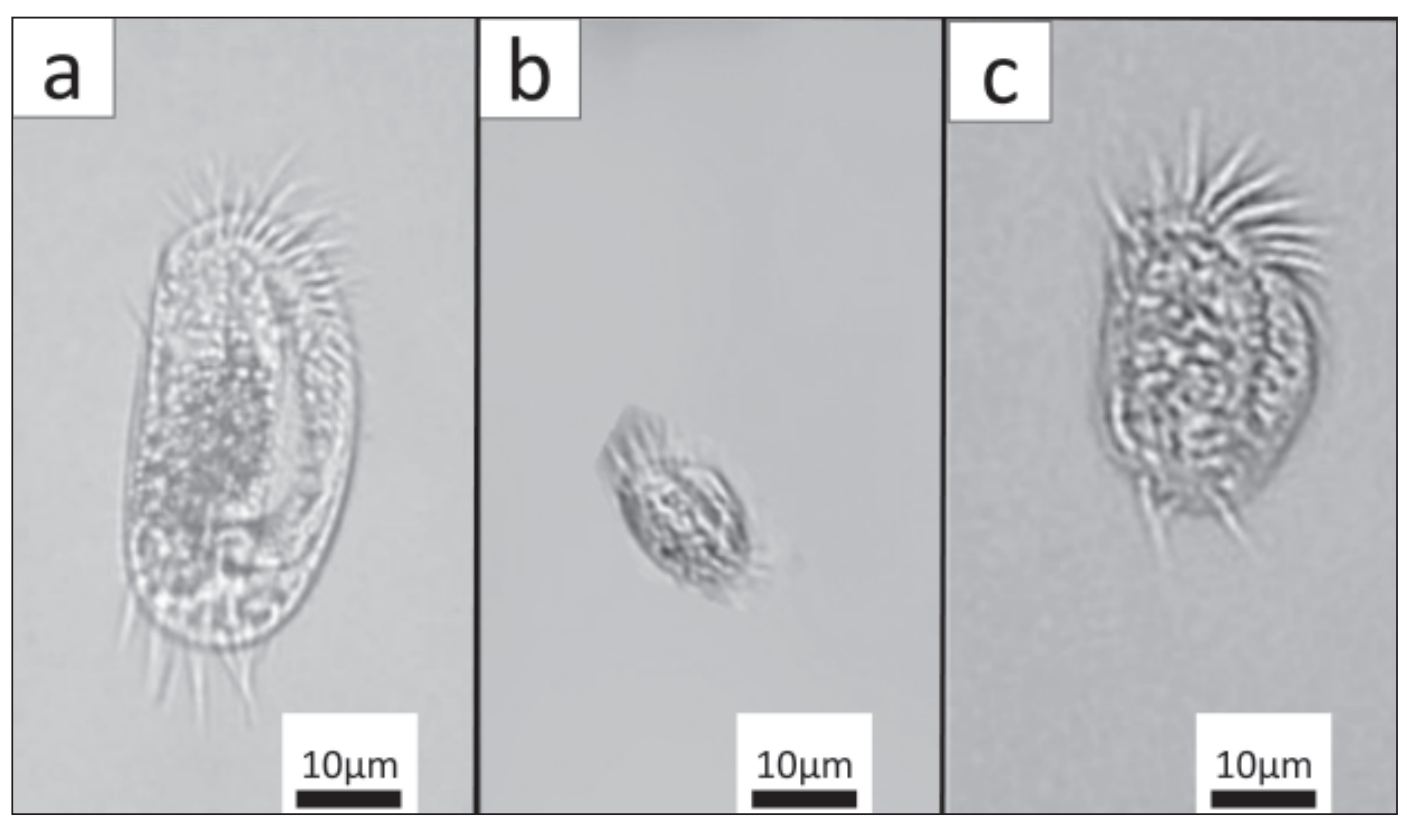

Fig. 2. Light microscope photographs of adult cells of each species. (a). Euplotes sp. 1, (b). Euplotes sp. 2, (c). Euplotes encysticus. Scale bar: $10 \mu \mathrm{m}$.

three species of Euplotes were observed, with approximate lengths as follows: Euplotes sp. 1,80 $\mu \mathrm{m}$; Euplotes sp. 2, $40 \mu \mathrm{m}$, and Euplotes encysticus $60 \mu \mathrm{m}$ (Figure 2). One-way ANOVA for densities showed significant differences on day 3 , and posthoc Tukey-Kramer test showed a significantly higher density at $10 \%$ than at $30 \%$. Densities on days 2, 4 and 6 were not significantly different. In summary, the density was the highest on day 3 in the water with the lowest salinity level.
The density of Euplotes spp. in the protozoa culture water at different salinity levels with elapsed days was observed (Figure 3). The Euplotes spp. peaked on day 3 and then decreased. One-way ANOVA showed significant differences on day 3 , and post-hoc Tukey-Kramer test showed a significantly higher density at $10 \%$ than at $30 \%$. There were no significant differences on other days. Again, the density was the highest on day 3 in the water with the lowest salinity level. The density of 


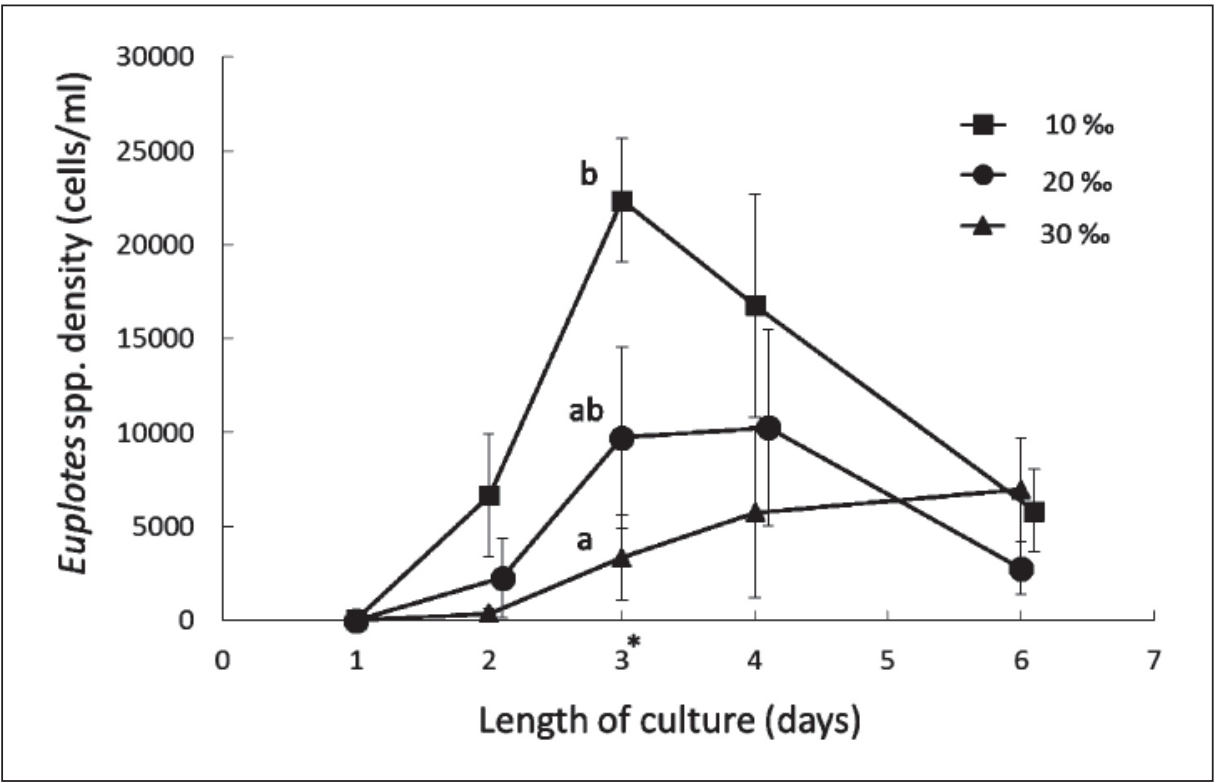

Fig. 3. Euplotes spp. densities (cell $\mathrm{ml}^{-1}$ : mean $\pm \mathrm{SE}$ ) in protozoa culture water (Fig. 1) at different salinity levels with elapsed days. ${ }^{*}$ Different lowercase letters indicate a significant difference on day 3.

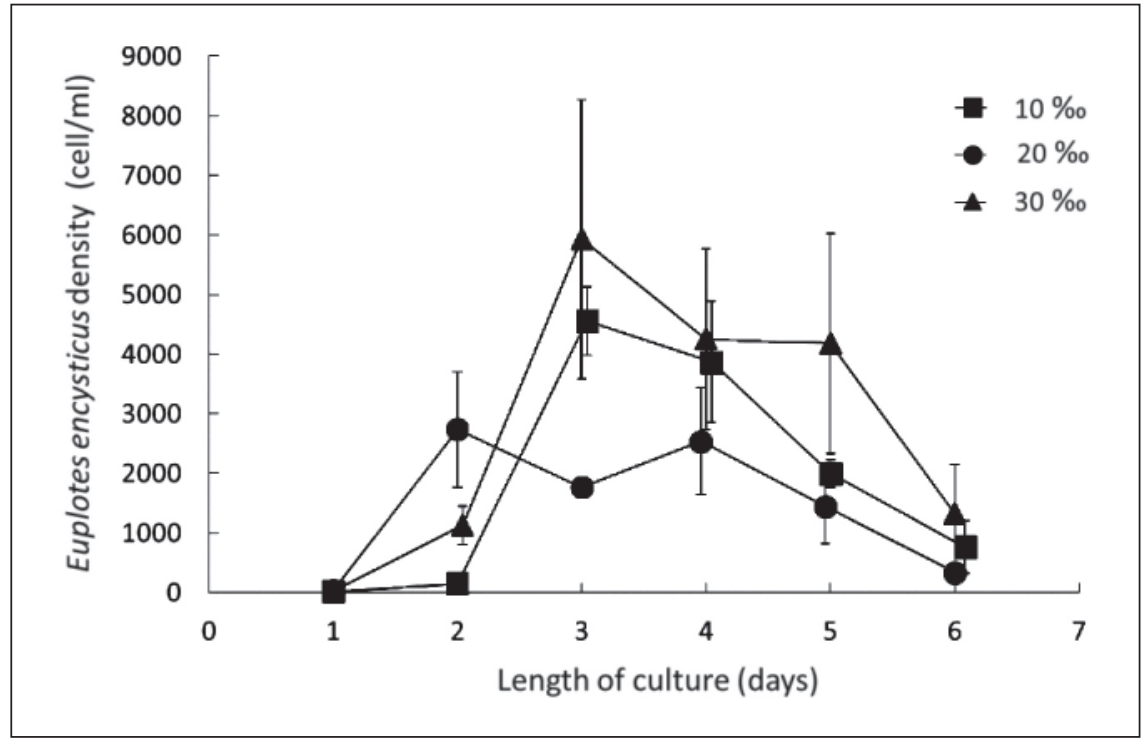

Fig. 4. Euplotes encysticus densities (cell $\mathrm{ml}^{-1}$ : mean $\pm \mathrm{SE}$ ) at different salinity levels with elapsed days.

Euplotes encysticus in the monoculture at different salinity levels with elapsed days was recorded (Figure 4). Peak densities were 3000-6000 cells $\mathrm{ml}^{-1}$. There were no significant differences between different salinity levels on all days.

\section{DISCUSSION}

In the present study, protozoa densities at different salinity levels were mostly not significant over the culture period except day 3 (Figure 1). However, the density in the lower salinity (10\%) group was significantly higher than that in the higher salinity group $(30 \%)$ on day 3. Euplotes spp. densities in the protozoa culture container also showed mostly no significant differences; however, the density in the lower salinity group $(10 \%)$ was significantly higher than that in the higher salinity group $(30 \%)$ day 3 . The E. encysticus monoculture results showed no significant density differences between different salinity levels over the culture period. 
Generally, rotifers are also cultured in water with a low salinity level (7\%-20\%) (Koiso \& Hino, 2001). The differences in the growth of protozoa at different salinity levels could be due to the difference in the osmotic pressure of ciliates cell fluid. The study regarding osmoregulation in marine ciliated protozoa is limited. Kaneshiro et al. (1969) addressed this topic in the marine ciliate Miamiensis avidus; the internal salinity of this ciliate is considerably lower than that of seawater, and the salinity of cell fluid changed when the salinity of the culture water was changed. The results of the current study regarding the effects of the salinity level of culture water are unclear, possibly as a result of the protozoa adapting to the salinity level of their environment, although we can conclude that a lower salinity level is most suitable for culturing protozoa.

Previous studies have used various methods for the culturing of ciliated protozoa. For example, Cortes and Tsuzuki (2012) cultured Euplotes sp. using yeast, Culture Selco and microalgae, and results showed a maximum density $(15,000,11,300$ and 237 cells $\mathrm{ml}^{-1}$, respectively) on day 6 . Rhodes and Phelps (2006) described a culture method for the ciliate Fabrea salina using the algae Isochrysis and Rhodomonas, achieving densities of 50-200 cells $\mathrm{ml}^{-1}$. However, the methods used in the present study produced a maximum density of 30,00040,000 cells $\mathrm{ml}^{-1}$ by day 3 and in lesser time than that observed in previous studies. The density achieved was high enough for rearing fish larvae. Ciliated protozoa $E$. encysticus also achieved a high density, i.e., a maximum of 5000-6000 cells $\mathrm{ml}^{-1}$. Mukai (unpublished data) succeeded in rearing larvae of Asian seabass (Lates calcarifer) and brown marbled grouper (Epinephelus fuscoguttatus) using the ciliated protozoa. Although further studies regarding nutritional composition of cultured ciliates are necessary, the present study method is suitable for culturing a starter diet for marine fish larvae.

The sizes of ciliated protozoa in this study were $<100 \mu \mathrm{m}$, which was smaller than the other types of live feed such as rotifers, and thus more suitable as a live starter diet for the larvae of fish species with small mouth openings that typically select food species $<100 \mu \mathrm{m}$. The optimum prey size for marine fish larvae is considered to be approximately $25 \%$ of the mouth width at first feeding, and the mouth width of early-stage larvae is typically 200-300 $\mu \mathrm{m}$ (Tucker, 1998). For example, Asian seabass selected 40-60 $\mu \mathrm{m}$ microcapsules when offered, those sized 15-150 $\mu \mathrm{m}$ (Walford et al., 1991). The three Euplotes spp. observed in the current study range in dimensions from 40 to $80 \mu \mathrm{m}$, suggesting that all of them are suitable to be used as live starter feed (Euplotes sp. 2 was identified as E. encysticus by molecular methods) (Mu'izzuddin-Afandi et al.,
2018). Especially, E. encysticus moved actively in the water such as rotifers, so it would be suitable live feed for fish larvae.

The culture method in the current study is simple, and the density of protozoa achieved is suitable for feeding fish larvae. Previous studies report the use of ciliated protozoa to culture marine fish larvae; for example, Rhodes and Phelps (2006) studied larval rearing of red snapper (Lutjanus campechanus) using both copepod nauplii and ciliated protozoa, achieving success. Nagano et al. (2000a) studied larval rearing of the grouper Hyporthodus septemfasciatus using Euplotes spp. They concluded that ciliated protozoa were an important food source, bridging the gap until the fish larvae were ready to eat copepod nauplii. Groupers, Napoleon wrasse and snappers have small bodies and small mouth openings in the early larval stage (Doi et al., 1997a; Nagano et al., 2000a; Hagiwara et al., 2014). These fish are commercially important in tropical regions. Fish reproducing in high temperature conditions produces a large number of small size eggs (Bone et al., 1996). Therefore, seed production of fish such as groupers and snappers is more difficult in tropical regions than in temperate regions due to the lack of suitable live starter feed in the tropical regions. In tropical regions, important marine finfish larvae, such as those of grouper and snapper species, have small bodies and small mouth openings at onset of feeding (Doi et al., 1997b). In high temperature conditions, fish produce small diameter eggs (Bone et al., 1996). Japanese anchovy (Imai, 1987) and Japanese flounder (Kurita et al., 2011) showed this tendency. Genus Epinephelus species are distributed from tropical to temperate region. It is supposed that these species comparatively smaller eggs in tropical region. This suggests that ciliated protozoa would be suitable as starter live feed for aquaculture in tropical regions.

In this study, protozoa culture was used with seawater having different salinity levels. Euplotes spp. and Uronema were included in the culture water. Euplotes spp. are useful, however Uronema $\mathrm{sp}$. is considered to be a parasitic protozoa, and cause disease to fish (Crosbie \& Munday, 1999). Therefore when the sea water that includes protozoa such as Uronema spp., monoculture method for objective ciliated protozoa e.g. Euplotes spp. would be recommended. Early-stage larvae consume protozoa in nature, and protozoa are reported to be a suitable live feed for fish larvae (Figueiredo et al., 2007; Montagnes et al., 2010; Zingel et al., 2012). If we consider the size and the achievable densities of cultured ciliated protozoa, it could be suitable live feed for fish larvae. Mass culture using the current study method and these nutrients are relatively straightforward. Therefore, cultured 
ciliated protozoa have high potential to be used as live feed for larvae of commercially farmed fish species in tropical regions.

\section{CONCLUSION}

In the present study, mostly there were not significant differences, however, the protozoa density in the lower salinity $(10 \%)$ group was significantly higher than that in higher salinity group $(30 \%)$ on day 3. Euplotes spp. also showed that the density in the lower salinity group (10\%) was significantly higher than that in higher salinity group $(30 \%)$ day 3 . The E. encysticus monoculture results showed no significant differences over the culture period.

\section{ACKNOWLEDGEMENTS}

We express sincere thanks to Miss Nur Al Mardhiah Yazid for the technical assistance. This study was supported by Fundamental Research Grants (FRGS/ 2/2014/SG03/ UIAM/02/2 and FRGS/1/2017/ STG03/UIAM/02/1) from Ministry of Higher Education Malaysia.

\section{REFERENCES}

Bone, Q., Marshall, N.B. \& Blaxter, J.H.S. 1996. Biology of Fishes. Chapman \& Hall, London. $332 \mathrm{pp}$.

Corliss, J.O. 1979. The Ciliated Protozoa: Characterization, Classification and Guide to the Literature, $2^{\text {nd }}$ editions. Pergamon Press, Oxford. 472 pp.

Cortes, G.F. \& Tsuzuki, M.Y. 2012. Effect of different live food on survival and growth of first feeding barber goby, Elacatinus Figaro larvae. Aquaculture Research, 43: 831-834.

Crosbie, P.B.B. \& Munday, B.L. 1999. Environmental factors and chemical agents affecting the growth of the pathogenic marine ciliate Uronema nigricans. Disease Aquaculture Organisms, 36: 213-219.

Curds, C.R. \& Vandyke, J.M. 1966. The feeding habits and growth rates of some fresh-water ciliates found in activated-sludge plants. Journal of Applied Ecology, 3: 127-137.

Das, P., Mandal, S.C., Bhagabati, S.K., Akhtar, M.S. \& Singh, S.K. 2012. Important live food organisms and their role in aquaculture. In: Frontiers in Aquaculture. M. Sukham (Ed.). Narendra Publishing House, Delhi. pp. 69-86.
Doi, M., Ohno, A., Kohno, H., Taki, Y. \& Singhagraiwan, T. 1997a. Development of feeding ability in red snapper Lutjanus argentimaculatus early larvae. Fisheries Science, 63: 845-853.

Doi, M., Ohno, A., Taki, Y., Singhagraiwan, T. \& Kohno, H. 1997b. Nauplii of the calanoid copepod, Acartia sinjiensis as an initial food organism for larval red snapper Lutjanus argentimaculatus. Aquaculture Science, 45: 31-40.

Figueiredo, G.M., Nash, R.D.M. \& Montagnes, D.J.S. 2005. The role of the generally unrecognized microprey source as food for larval fish in the Irish Sea. Marine Biology, 148: 395-404.

Figueiredo, G.M., Nash R.D.M. \& Montagnes, D.J.S. 2007. Do protozoa contribute significantly to the diet of larval fish in the Irish Sea? Journal of the Marine Biological Association of the United Kingdom, 87: 843-850.

Fukami, K., Watanabe, A., Fuzita, S., Yamaoka, K. \& Nishijima, T. 1999. Predation on naked protozoan microzooplankton by fish larvae. Marine Ecology Progress Series, 185: 285-291.

Hagiwara, A., Wullur, S., Marcial, H.S., Hirai, N. \& Sakakura, Y. 2014. Euryhaline rotifer Proales similis as initial live food for rearing fish with small mouth. Aquaculture, 432: 470-474.

Imai, C. \& Tanaka, S. 1987. Effect of sea water temperature egg size of Japanese anchovy. Nippon Suisan Gakkaishi, 53: 2169-2178.

Kaneshiro, E.S., Dunham, P.B. \& Holz, J.R.G.G. 1969. Osmoregulation in a marine ciliate, Miamiensis avidus, I. regulation of inorganic ions and water. Biological Bulletin, 136: 63-75.

Kitani, K. 1989. Cultivation and utilization of a ciliated protozoa Infusoria. Aquacultue Science, 36: 307-320.

Koiso, M. \& Hino, A. 2001. Effects of salinity on population growth, nutritive value and feeding cost of the enriched rotifer, Brachinous plicatilis. Aquaculture Science, 49: 41-46.

Kurita, Y., Fujinami, Y. \& Amano, M. 2011. The effect of temperature on the duration of spawning markers - migratory-nucleus and hydrated oocytes and postovulatory follicles in the multiple-batch spawner Japanese flounder (Paralichthys olivaceus). Fishery Bulletin, 109: 79-89.

Madhu, K. \& Madhu, R. 2014. Captive spawning and embryonic development of marine ornamental purple firefish Nemateleotris decora (Randall \& Allen, 1973). Aquaculture, 424-425: 1-9. 
Mu'izzuddin-Afandi, M., Waludin, S.N., Mohammad-Noor, N., Phang, I.C., Kadowaki, S. \& Mukai, Y. 2018. Identification of Euplotes encysticus a starter live feed for marine fish larvae based on morphology and molecular sequences. Malaysian Applied Biology Journal, 47: 175-180.

Mukai, Y., Sani, M.Z., Mohammad-Noor, N. \& Kadowaki, S. 2016. Effective method to culture infusoria, a highly potential starter feed for marine finfish larvae. International Journal of Fisheries and Aquatic Studies, 4: 124-127.

Mieczan, T. 2007. Size spectra and abundance of planktonic ciliates within various habitats in a macroohyte-dominated lake (Eastern Poland). Biologia Blatislava, 62: 189-194.

Montagnes, D.J.S., Dower, J.F. \& Figueiredo, G.M. 2010. The protozooplankton Ichtyoplankton trophic link: an overlooked aspect of aquatic webs. Journal of Eukaryotic Microbiology, 57: 223-228.

Nagano, N., Iwatsuki, Y., Kamiyama, T., Simizu, H. \& Nakata, H. 2000a. Ciliated protozoans as food for first-feeding larval grouper, Epinephelus septemfasciatus: laboratory experiment. Plankton Biology and Ecology, 47: 93-99.

Nagano, N., Iwatsuki, Y., Kamiyama, T. \& Nakata, H. 2000b. Effects of marine ciliates on survivability of the first-feeding larval surgeonfish, Paracanthurus hepatus: laboratory rearing experiments. Hydrobiologia, 432: 149157.
Olivottoa, I., Zenobia, A., Rollob, A., Migliarinia, B., Avellab, M. \& Carnevalia, O. 2005. Breeding, rearing and feeding studies in the cleaner goby Gobiosoma evelynae. Aquaculture, 250: $175-182$.

Rhodes, M.A. \& Phelps, R.P. 2006. Ciliated protozoans as alternative live food for first feeding red snapper, Lutjanus campechanus, larvae. In: Proceedings of 57 Annual Gulf Caribbean Fisheries Institute. R.L. Creswell (Ed.). pp. 963-973.

Tucker, J.R.J.W. 1998. Marine Fish Culture. Kluwer Academic Publishers, Massachusetts. 750 pp.

Winter, F. \& De Persoone, G. 1975. Preliminary experiments with the ciliate Fabrea salina as a potential live food for mariculture. In: $10^{\text {th }}$ European Symposium on Marine Biology, Ostend, Belgium. pp. 37-48.

Walford, L., Lim, T.M. \& Lam, T.J. 1991. Replacing live foods with microencapsulated diets in the rearing of seabass (Lates calcarifer) larvae: do the larvae ingest and digest protein-membrane microcapsules? Aquaculture, 92: 225-235.

Zingel, P., Paaver, T., Karus, K., Agasild, H. \& Noges, T. 2012. Ciliates as the crucial feed source of larval fish in a shallow eutrophic lake. Limnology and Oceanography, 57: 1049-1056. 
